\title{
Adapting the Delivery of Functional Family Therapy Around the World During a Global Pandemic
}

\author{
Michael S. Robbins ${ }^{1,2}\left[\right.$ Helen Midouhas ${ }^{1}$
}

Received: 8 October 2020 / Accepted: 7 April 2021 / Published online: 26 April 2021

(c) This is a U.S. government work and not under copyright protection in the U.S.; foreign copyright protection may apply 2021

\begin{abstract}
This paper describes accommodations that were implemented to support the global delivery of Functional Family Therapy (FFT) in community-based settings during COVID-19. FFT is an evidence-based family therapy that has been used with youth and families in juvenile justice, child welfare, mental health, and school settings. FFT LLC, an organization that disseminates FFT into community settings, currently supports over 350 community-based agencies that deliver FFT globally. Using the implementation framework articulated by Fixsen et al. (Implementation research: a synthesis of the literature, University of South Florida, Louis de la Parte Florida Mental Health Institute, The National Implementation Research Network, 2005), this paper describes key sets of implementation drivers (i.e., competency, organizational, and leadership) that facilitated the accommodations made to meet the challenge of maintaining fidelity to FFT as training and clinical services were transitioned to online platforms. To evaluate if accommodations were associated with fidelity and outcomes, descriptive information about treatment delivery and basic outcomes (e.g., therapist fidelity to model, youth behavioral outcomes) from March 15, 2020, to September 1, 2020 were compared to the same period from 2019. During 2020, FFT LLC supported therapists who served over 11,000 families and conducted over 35,000 tele-sessions with families. Overall, results showed similar completion rates ( $79 \%$ vs. $75 \%$ ), therapist fidelity (3.77 vs. 3.94), and therapist-reported outcomes in 2019 and 2020 (respectively), suggesting that delivering the FFT model can be implemented with fidelity using teletherapy formats.
\end{abstract}

Keywords Family therapy $\cdot$ Tele-sessions $\cdot$ Pandemic $\cdot$ Implementation $\cdot$ International

\section{Introduction}

Implementing an evidence-based psychotherapy practice model with fidelity in community settings is challenging under ideal circumstances. The outcomes achieved in controlled trials are hard to replicate in community settings (Henggeler et al., 1999). It can take years for a practice model to achieve comparable real-world effects (Yuan et al., 2010). Over the past two decades, the development of implementation frameworks that guide the factors associated with successful implementation (e.g., implementing the practice model with fidelity and comparable outcomes to prior research) has helped inform dissemination efforts and enhanced implementation outcomes (Bertram et al.,

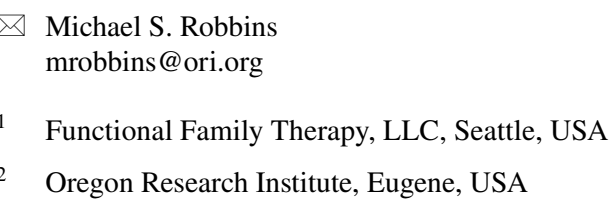

2015). One of the most notable examples of disseminating evidence-based practice models into community settings is implementing evidence-based family therapy models for youth with behavior problems, such as delinquency, substance use, and aggression/violence. Several family-based models have been widely disseminated in community settings, including Functional Family Therapy (FFT; Alexander \& Parsons, 1982; Alexander et al., 2013) and Multisystemic Therapy (Henggeler et al., 2009).

In March 2020, the landscape for implementing practice models in real-world settings became anything but ideal. The novel coronavirus-2019 (COVID-19) created an entirely new set of circumstances and barriers to implementation that emerged virtually overnight. Youth, family members, therapists, and community stakeholders were impacted at every possible level (i.e., physical, psychological, and financial). [Note: The term therapist is used to describe the provider of clinical services. Therapists are licensed or licensed eligible providers that typically have a background in psychology, counseling, social work, or family therapy.] A "new normal" 
that included social distancing and mandatory quarantines forced a new set of practice considerations onto therapists, systems, and training organizations. Face-to-face training and clinical services were no longer an option in most circumstances. For family-based treatments that focused on influencing the relational space between people, this shift in practice was both immediate and profound, and-unfortunately - the lessons from over 20 years of community-based implementation and research provided little to no guidance about how to effectively address these new factors.

This paper describes how an organization that provides training and consultation in FFT (FFT LLC; www.FFTLLC. com) supported therapists, agencies, and communities respond to the challenge of COVID-19. The first section briefly presents features of a well-established implementation framework (Fixsen et al., 2005) to provide a context for understanding and summarize the types of implementation strategies used to support FFT delivery. The second section provides a brief overview of FFT and a description of the standard training parameters used by FFT LLC. The third section presents how training, consultation, and clinical implementation were altered to support the pivot from face-to-face sessions to tele-services. Finally, in the fourth section, descriptive information is provided about therapist fidelity and primary clinical outcomes to illustrate these implementation accommodations' impact. Families served from March 15, 2020, to September 1, 2020, were compared to families served during the same period in 2019. During 2020 , over 11,000 families received FFT through the provision of over 35,000 tele-sessions. Overall, similar completion rates, therapist fidelity, and therapist-reported outcomes in 2019 and 2020 were observed.

\section{Implementation Framework}

This paper uses the implementation framework developed by Fixsen et al. (2005) to organize the accommodations made to support FFT delivery during the early stages of COVID19. This framework postulates that the primary focus of any community-based implementation of established practice models is to ensure the competent delivery (i.e., fidelity) of the intervention to the population it was intended to serve. Overall, success includes both high-quality implementation as well as the achievement of relevant outcomes. This framework recognizes that putting a practice model on the ground in real-world settings is not a simple process that is limited to teaching providers a new technique or set of strategies. It is a complex process that requires systemic and organizational change and support. Fixsen et al. (2005) describe three broad sets of "implementation drivers" critical for successful implementation and innovation in community-based settings: Competency, Organization, and Leadership. Each set includes multiple drivers that are essential for successful implementation.

Competency drivers capture some of the most proximal strategies for ensuring fidelity and include coaching, training, and staff selection. The competency drivers reflect a central focus on fidelity first. They represent the range of direct training and coaching necessary to help therapists learn interventions and then practice the interventions with increased sophistication, confidence, and competence over time. The training characteristics tend to be fixed (e.g., specific training activities delivered at a certain time), whereas coaching is often more dynamic and tailored to a therapist's and organization's individual needs. Careful attention is given to selecting staff with values, beliefs, and experience that suggest a readiness to provide the practice model. The ongoing performance assessments inform the coaching process of therapist delivery of the practice model. These assessments help identify strengths and areas of concern. Staff selection is not simply about the service providers. It also includes selecting administrators, managers, and supervisors with the skills sets (or capabilities to develop the skill sets) necessary to direct and support implementation.

Organizational drivers capture essential contextual influences on the successful adoption of an intervention or innovation. Three interacting sets of organizational drivers are critical in supporting the effective delivery of the practice model with fidelity, including systems-level interventions, facilitative administration, and data systems' availability to support decision-making. These interventions address external barriers, such as reimbursement rates, local or state regulations, referral issues. Facilitative administration involves direct support of therapist practice within an organization (e.g., caseload sizes, availability of resources, removing additional responsibilities). Decision support data systems are critical for monitoring fidelity and outcomes on an ongoing basis. The effective utilization of data is most successful when used in a supportive rather than punitive manner. Facilitative administrators and other leaders in the implementation process should continuously use data to support therapists by capitalizing on improvements, removing barriers to implementation, and developing and implementing tailored improvement plans for therapists, supervisors, and administrators/managers.

Leadership drivers include both technical and adaptive leadership. The leadership styles are not intended to reflect individuals' static or fixed characteristics but rather capture how leaders can respond and develop innovative solutions to challenges. For example, technical leadership is necessary when there is consensus about a problem or solution. It involves consistent and effective management to ensure that plans are put in place and supported. In contrast, adaptive leadership is necessary when the challenges are less clear, and there might not be a consensus about how to proceed. In 
these circumstances, adaptive leadership is needed to facilitate a process with key stakeholders and staff to arrive at innovative solutions to unique situations. This process builds on a foundation of authentic relationships with stakeholders developed and nurtured at every stage of the implementation process.

The implementation drivers are dynamic and synergistic, interacting to produce innovation in the face of change. To meet the changes encountered during the early stages of COVID-19, the training organization, community-based therapists, agencies, and community stakeholders had to embrace an openness to innovation and a willingness to create change. Technical and adaptive leaders' presence was critical for ensuring that accommodations were immediate and responsive to a swiftly changing context. For each of these drivers, maintaining fidelity guided all accommodations that were implemented to ensure that therapists could deliver FFT to families and youth in need.

\section{Overview of FFT}

FFT (Alexander \& Parsons, 1982; Alexander et al., 2013) integrates systems and cognitive-behavioral interventions to address a range of youth effectively (e.g., aggression, violence, criminal, substance use, anxiety, depression) and family (e.g., parent distress, sibling criminal behavior, family conflict) problems (Robbins et al., 2016). Treatment is provided in 12-14 sessions on average and up to 30 sessions with challenging cases and is expected to be relatively brief (e.g., 3-5 months). Sessions include multiple family members and are usually conducted in the family home and are usually 45-60 min.

Over the first 30 years of development (1970 to 2000), FFT was implemented and evaluated with youth and families from diverse settings (e.g., juvenile justice, school, mental health, child welfare) as both a preventive and treatment approach. Over the past two decades (2000 to 2020), FFT LLC has supported the implementation of the FFT model as well as promising variations, including FFT-Child Welfare (FFT-CW ${ }^{\circledR}$; Turner et al., 2017), FFT-Gangs (FFT-G ${ }^{\circledR}$; Gottfredson et al., 2018), and FFT-Probation Services (FFP ${ }^{\circledR}$; Darnell \& Schuler, 2015). Annually, FFT LLC supports over 3000 therapists, supervisors, and administrators/managers implementing FFT, FFT-CW ${ }^{\circledR}$, FFT-G $^{\circledR}$, and FFP ${ }^{\circledR}$ with more than 20,000 youth and families in 10 countries. The strategies that FFT LLC uses have been informed by more than 20 years of implementation experience and the more extensive literature on implementation research and practice. For example, in a large-scale community implementation of FFT-CW ${ }^{\circledR}$ (Turner et al., 2017), the implementation framework developed by Fixsen et al. (2005) was incorporated into all levels of implementation by the local child welfare system.

The FFT LLC training approach is designed to assist therapists, supervisors, and administrators/managers from community-based agencies and governmental organizations in becoming proficient in implementing FFT with fidelity and effectiveness. [Note: Due to the consistency in training across FFT and the model variations, the term "therapists" is used throughout the remainder of the paper.]

Before starting any training, all prospective sites go through an extensive readiness process. This process includes conversations about the "fit" between organizational values and the FFT model, organizational characteristics necessary to support successful implementation, appropriate workforce availability, referral considerations, relationships with critical community stakeholders, and long-term sustainability. The final step in the readiness process is a Stakeholder Meeting led by an FFT LLC implementation expert to introduce the model to stakeholders and discuss implementation characteristics formally. A primary focus of the pre-implementation activities is to begin developing the requisite organizational and leadership skills within agencies and the community to drive the competent implementation of FFT.

Training of therapists is completed in three stages (see Table 1). The activities of Stage 1 are designed to build therapist fidelity to the FFT model and include five onsite trainings, use of a web-based Client Service System (CSS), and a weekly consultation led by an FFT LLC expert. The weekly consultation with the FFT LLC expert involves video conferencing; however, the therapists are expected to be together for this meeting to facilitate cohesion and learning from each other's cases. The therapists are also expected to meet on their own once per week in a face-to-face peer group meeting to develop their model skills further. In this sense, sites are actively engaged to provide support and guidance and remove barriers to successful implementation (e.g., decrease redundancy in the documentation). During Stage 1, the FFT LLC expert works closely with site administrations to further develop organizational and leadership skills to ensure the site's long-term sustainability. For example, a common area of discussion is relationship building with key community stakeholders to ensure there is a sufficient number of appropriate referrals to support a practice over time. The continuous use of data derived from the CSS is a crucial driver for training and consultation and for developing and refining implementation processes with administration and community stakeholders.

At the end of Stage 1, one of the therapists is identified as the "site supervisor." They attend an offsite externship (three, 3-day trainings). The activities of Stage 2 are designed to develop a strong site supervisor who can assume most of the activities that were delivered by the 
Table 1 Stages of FFT training: standard and modified

\begin{tabular}{lll}
\hline Stages & Standard delivery & Modifications for COVID-19 \\
\hline Stage 1 & & \\
Pre-stakeholder meeting & In person-On-site & Webinar [Started May 2020] \\
Pre-CSS training & In person-On-site & Webinar [Started March 2020] \\
Initial clinical training & In person-On-site & Webinar [Started March 2020] \\
Second clinical training & In person-On-site & Webinar [Started March 2020] \\
Follow-up training & In person-On-site & Webinar [Started March 2020] \\
Weekly consultation & Webinar & Webinar [Started March 2020] \\
Weekly peer group & In-person-On-site & Webinar [Started March 2020] \\
Supervision & In person-Off-site & Webinar [Started March 2020] \\
Externship & In person-Off-site & Webinar [Started September 2020] \\
Stage 2 & & \\
Supervisor training & In person-Off-site & Webinar [Started March 2020] \\
Bi-weekly consultation & Webinar & Webinar [Started March 2020] \\
Site visit & In person-On-site & Webinar [Started March 2020] \\
Stage 3 & & \\
Monthly consultation & Webinar & Webinar [Started March 2020] \\
Site visit & In person-On-site & Webinar [Started March 2020] \\
Miscellaneous & & \\
Replacement training & In person-Off-site & Webinar [Started March 2020] \\
\hline
\end{tabular}

FFT LLC expert in Stage 1. These activities include conducting weekly supervision with therapists, monitoring therapist progress through the use of data, interfacing with agency leaders (including managers that are directly overseeing the implementation of FFT), and interfacing with key community stakeholders as needed. The training activities in Stage 2 include two offsite supervisor trainings, one onsite training, and bi-weekly consultations with the site supervisor. FFT LLC experts continue to provide guidance and support through bi-weekly calls with the site supervisor. The calls focus on several areas: nurturing onsite organizational processes and leadership skills, developing skills for running reports in the CSS and using the data to support therapist development, and facilitating supportive practices with community stakeholders.

The activities of Stage 3 are designed to develop the skills of the site supervisor further. FFT experts conduct a monthly meeting with the site supervisor and an annual site visit. As in Phase 2, the focus of Phase 3 is to continue monitoring evidence that the FFT model is being implemented with fidelity and effectiveness and to developing and supporting organizational and leadership drivers that are essential for the implementation of the FFT model. In the last decade, the FFT LLC training model's effectiveness is evidenced in over 20 peer-reviewed evaluations representing real-life clinical work in 6 countries and 7 U.S. states (Functional Family Therapy [FFT], 2021).

\section{Pivoting to Online Work}

As noted above, most therapy sessions are conducted face to face with families in their homes and are approximately 45 to $60 \mathrm{~min}$. The location of treatment sessions is not fixed, but it is based on convenience for the family. Historically, FFT LLC has permitted the use of teletherapy services (e.g., video conferencing) in exceptional circumstances. For example, in one state-wide initiative that included many rural locations, tele-sessions were supported as an option when there were significant weatherrelated barriers to meet in person, but this option was rarely used. Thus, 2020 represented the first consistent and wide-spread utilization of tele-services by FFT LLC.

In February 2020, FFT LLC was concerned about the potential implications of COVID-19. Still, like most, the scope of what a global pandemic meant for their community-based partnerships and clinical work with families was not fully realized. Organizationally, FFT LLC had never discussed or developed a response to this kind of scenario. Moreover, the uncertainty and inconsistencies in forecasts at the national and global levels provided little guidance or direction. By early March, it was clear that a coherent organizational response around travel, consultation and training, and service delivery was necessary. It was also recognized that any response needed to place the commitment to practicing FFT with fidelity (i.e., competency) to its core principles at the center of all decisions. 
On March 8, FFT LLC distributed a letter providing specific recommendations and guidance to sites, including (a) support for the clinical delivery of sessions via tele-services in later phases of treatment, (b) support for therapists joining weekly consultation sessions via a web-based platform rather than in person, (c) flexibility about scheduled onsite training (e.g., delaying training to a later date), and (d) a commitment to continue offsite training activities for community agencies that wanted to proceed with in-person training. Given the lack of certainty in transmission and social guidelines at this early stage in the pandemic response, recommendations were limited to cover the time period up to April 15, 2020.

In these early days of developing a coherent response, the FFT LLC leadership team reviewed information daily. For example, international partners regularly provided information about updated travel guidelines and mandatory quarantines. The leadership team decided to switch from a flexible approach to a more fixed set of recommendations because it was determined that maintaining a flexible approach to working with sites would lack consistency, and it would become unwieldy in the face of the variability in how local communities, states, and countries were responding. As such, on March 20, 2020, a revised letter was issued that (a) extended the support for delivering FFT via tele-sessions over all phases of treatment, (b) increased the timeframe for supporting tele-services to the end of May 2020, and (c) suspended all FFT-related travel until the end of April 2020. Suspending all travel meant that training activities were either going to be delayed for some time until it was deemed safe or that the training would be delivered via a webinar format. This specificity helped organizations take the steps needed to help institute changes required to continue to deliver services and provided them with support for communicating to stakeholders that the changes met fidelity requirements.

Letters were updated monthly from March to June 2020 and every three months thereafter. The updates' primary purpose was to increase the timeframe for supporting the delivery of tele-sessions through the end of 2020. This outreach remained relevant for sites that needed formal communication from the training organization, noting the approval of tele-sessions. It was also applicable for sites returning to more restrictive "lockdown" levels due to the virus's outbreak.

\section{Pivoting to Webinar-Based Training and Consultation}

The most important shift in the support of communitybased agencies was moving to webinar-based training. Initially, delaying the training until later dates when it would be safe to travel was considered. However, this was viewed to be inappropriate because sites were continuing to work with families. It was also clear that the length of time that COVID-19 would disrupt travel and in-person work would be significant. As such, all follow-up trainings (Stage 1), supervisor trainings (Stage 2), site visits (Stage 3), and replacement trainings were converted to a webinar format. Modified training activities are presented in Table 1.

The only training component that did not immediately shift to webinar was the in-person externship (Stage 1). Recently, FFT LLC (September 2020) started conducting virtual externships. The training of new community-based agencies was initially delayed; however, as it became clear that systems were continuing to support training for the practice model, FFT LLC also shifted all pre-implementation work, including stakeholder meetings, to a web-based format.

The primary focus of web-based training was on ensuring competency by replicating as many in-person training experience elements as possible. This replication was achieved by reviewing every training protocol's goals and breaking down the key components of training. Replicating the content of the training was a simple task. New materials did not need to be created; however, it was necessary to remove some of the slides' redundancy to increase the training's efficiency. The 2-day format was adjusted to include briefer presentations spread over several days. Over time, however, many community-based agencies preferred a format that resembled the standard, 2-day training.

\section{Key Drivers of Service Delivery}

Various changes were implemented to ensure that the FFT model was delivered to family members with fidelity. In the section below, the accommodations to deliver FFT services are organized into three areas: Creating and Supporting Resources for Tele-Services, Working Effectively with Community Stakeholders, and Specific Clinical Guidance. It is worth noting that the community agencies were at different implementation stages: Some had been providing FFT for two decades, while others were in their first year of implementation, and a number were even launched during 2020. As such, across communities, there was considerable variability in the leadership skills and systemic processes necessary to support implementation. Where relevant, information was provided about the individuals, organizations, and systems involved in providing direction and leadership.

\section{Creating and Supporting Resources for Tele-Services}

To support the transition to tele-services, the key leaders at FFT LLC immediately established communication loops within the organization and between the organization and community providers. As noted above, FFT LLC's 
leadership issued a monthly letter to provide direct support to agencies. The monthly letters were more than merely providing a supportive message. There was a central concern about how agencies would manage and sustain their programs as countries and communities began instituting restrictions in socialization. One overarching matter was a financial one, which drives all the other processes from service delivery, and training, to paying staff and keeping doors open. About $40 \%$ of the states where FFT is provided utilize government funding for reimbursement. Overnight, funders and managed care entities were turning to FFT LLC to provide guidance as well. A central question was whether FFT could be delivered with fidelity via a teletherapy format. This inquiry prompted the leadership at FFT, LLC, to add the option of "tele-session" to the web-based Client Service System (CSS) to capture data on various aspects of model delivery. The ability to track these sessions allowed timely feedback to funders to support this modality.

The impact of this guidance for community-based partners was immediate and positive. For example, the recommendations to support tele-services facilitated agency leaders to work with funding agencies to adapt the guidelines that would permit payment for FFT via telehealth platforms. This example represents the type of adaptive leadership that was required during this transition. In many areas, larger systems and stakeholders were not initially on board with transitioning therapeutic services to tele-platforms. Community agency leaders had to work collaboratively with key stakeholders to maintain continuity of services for youth and families. The transition to tele-services also required facilitative administration at local sites. Organizations needed to secure or redirect resources to support tele-services. This support included meeting basic requirements, such as providing equipment to therapists and families with laptops, smartphones, or tablets. It also involved developing agency policies to support tele-services (e.g., working from home policies, technological troubleshooting, and fail plans). Facilitative administration also involved creative strategies to fund these resources. For many organizations, facilitative administration was further enhanced through systems-level interventions targeting increased collaboration with community stakeholders to support tele-services by maintaining the model and providing tangible resources (computers, free Wi-Fi, community hot spots) to the families they referred. Many families did not have reliable internet access, and agencies/communities were creative to find ways to ensure that access was not an issue. Some agencies converted unused therapists mileage/travel budgets to pay for smartphones, tablets, and laptops. One agency created a local hotspot outside of their community center. Also, multiple communities provided free internet access to lower-income families.
Extensive coaching and support were provided to therapists to transition to tele-services by leadership at community agencies and FFT LLC experts. For example, for the latter, FFT LLC also distributed a weekly "Lessons Learned" document that provided therapists with specific recommendations about accommodations in implementation. The Lessons Learned focused extensively on how to competently deliver FFT via tele-session. Leaders from agencies and FFT LLC recognized the critical importance of attending to the personal impact of COVID-19 on therapists. Therapists themselves were struggling with the feeling that they were achieving less in this COVID-19 world; they felt less effective, less adequate, less confident, or less successful. Added to this was the reality that everyone struggled with the discomfort of not knowing what FFT "should" look like in the COVID world. There were more unknowns than knowns as this transition started. It was critical to acknowledge this experience at the therapist level (e.g., managing their stress and discomfort) and then extend it to their work with families who were also experiencing the same doubts about life during COVID-19. Consultation and supervision included more frequent conversations about therapist selfcare, including ideas for helping the therapists to manage new platforms of delivering services without becoming overwhelmed. Much of this support was simple and direct (e.g., encouraging therapists to scheduling breaks between sessions to avoid fatigue associated with video-based teleservices), but it also involved consistently attending to the stress that therapists experienced ongoing basis.

The shift to tele-services required additional training to ensure that therapists were proficient in the new technology. If therapists were expected to be competent in delivering the FFT model with fidelity over the internet, they also needed to develop competence in working with the new tele-platforms. The weekly "Lessons Learned" document provided such training by utilizing feedback from others familiar with the technology and sharing these competent strategies with all agencies. This document evolved based on input from therapists as their experience implementing tele-sessions with families. Initially, the document was heavily focused on preparing therapists for this change and included areas such as a) managing negative expectations (e.g., "This will not work."), b) addressing fear and anxiety, c) therapist attitude (e.g., "fake it til you make it," find a way to be yourself), d) humility (e.g., you do not need to be an expert in all things), e) practicing in supervision, f) managing stress (e.g., mindful practice for managing anxiety), and later g) managing computer fatigue. Later, the document evolved to help families who were not comfortable with the medium and more advanced techniques related to model phases.

FFT LLC added guidance about areas to consider when organizing sessions and introducing tele-services to families. This guidance included essential elements like internet 
access, equipment, camera placement, setting up and logging into a service, and general recommendations about the clinical work itself. For the latter, therapists reported that sessions felt slower, so recommendations were provided about how to "amp-up" energy, activity level, and animation. This focus on activity was necessary for therapists to put themselves into the family space and create a presence "in the room." One therapist expressed this sentiment by noting that "I'm learning that I set the tone."

One final part of organizing tele-services involved developing specific safety protocols to manage a variety of situations, such as a) family members argued and, in turn, shut down the link to the session, b) a family member was suicidal or homicidal, or c) there was suspected abuse or neglect. These included having back-up numbers for family members and direct communication about safety plans at the start of every session in high-risk families.

Facilitative leadership and systems-level interventions made it possible to provide the space for therapists to increase their proficiency in the use of tele-services by engaging in the types of activities described above. For example, many agencies altered their policies and productivity requirements to allow more time to complete initial paperwork and assessments.

\section{Working Effectively with Community Stakeholders}

The leaders at community agencies and FFT LLC recognized that the social restrictions during COVID-19 required accommodations at multiple levels, including the interface with community stakeholders. Ultimately, however, the community agencies' leaders facilitated the accommodations to the nature, frequency, and intensity of their work with community stakeholders. For example, the changes in social guidelines directly impacted interfacing with systems during the referral process. Most agencies had a mechanism for linking families to service, and initial contacts often occurred face to face. Other systems required that the initial contact includes a caseworker or probation worker as part of the referral process. In these situations, the communitybased agency needed to establish a new approach for case transfer. This process created an opportunity to strengthen relationships with referral sources and stakeholders to ensure an efficient and timely link to services. However, with more people involved in the process, it became easier for a family to "slip through the cracks" either intentionally by creating a chance to avoid services or unintentionally by not getting a family access to help for services. Building a direct communication channel with critical stakeholders helped to prevent this from occurring. For example, with high-risk cases being referred from child welfare systems, the more closely agencies worked with the referral system, the easier it was to manage initial risk. This relationship was critical because, in many circumstances, the child welfare system was unable to perform some of the necessary in-person regulatory activities (e.g., home visits), and FFT therapists were left carrying the responsibility for identifying and addressing risk. A close working relationship was particularly relevant in high-risk cases in which there is the potential for imminent risk, such as abuse, neglect, and family violence. Features of the relationship with helpful referral sources are:

(1) Clearly identified roles and responsibilities during the initial referral and engagement process

a. System maintaining responsibility for determining safety pre-intake and before engagement in services

b. System being available throughout the early sessions for assessment of emerging safety considerations

c. Agency/therapist responsible for engaging family members into treatment and then motivating them for change

(2) Clear and regular communication about:

a. The reason for the referral

b. Risk assessments

c. This communication is transparent to family members

Another accommodation during COVID-19 was that therapists found it necessary to access additional services much earlier in the treatment process, particularly for highrisk cases. This shift was not an adaptation of FFT since this was always a possibility based on a family's characteristics. What changed was the reduced availability of and contact with mandatory reporters lowered the threshold for the time therapists were willing to give to facilitate changes in family skills. Thus, rather than waiting for skills to develop and then generalizing the skills to new situations, therapists started to pull in services that might have been left to the final treatment sessions. For example, appropriate referrals might include a referral for psychiatric treatment for anxiety or depression, Alcoholics Anonymous, or bringing in extended family members to assist with childcare or household responsibilities.

Accessing external resources has been more challenging. As such, some of the recommendations involved a more long-term plan for accessing these resources once the restrictions on social contact were removed. This plan involved developing information packets with families and discussing steps for creating links. Therapists noted that discharging families was more challenging during COVID. This challenge was due to therapists being reluctant to close because the services that they had linked to in the past might not be available due to COVID-19 restrictions. The solution was 
to try to connect to natural support systems as much as possible (e.g., extended family members, neighbors, churches, friends), identify services using tele-platforms, and bolster plans with appropriate self-guided resources (e.g., links to online clinical practices). For some families, the reluctance to close was based on loneliness and uncertainty. Therapists were encouraged to be patient with discharging cases and to recognize that closure is a process, not a discrete event. As such, closure can be reached over multiple, shorter contacts with the family and then build in planned booster sessions after discharging the case.

\section{Specific Clinical Guidance}

The primary challenge in shifting to tele-services was to ensure that fidelity was maintained. This required responsive leadership at the training organization to quickly develop clinical implementation plans and communicate them to community agencies. This feedback was provided directly to the FFT experts who led the ongoing coaching of therapists serving youth and families. However, it is essential to note that these plans evolved based on therapists' experience and feedback about new challenges encountered and the strategies they found to be particularly useful in implementing the FFT model. Several examples of clinical accommodations are provided below.

Many therapists reported increasing the number of contacts per week and conducting shorter but more frequent sessions. In part, these changes were due to the new medium; however, this was also driven by new clinical challenges that were emerging during COVID-19 (see next section for more detail). This feedback was immediately incorporated into the Lessons Learned document and distributed to all agencies.

One of the ways in which an increase in contact has occurred is that therapists have used intake and other assessments as opportunities for face-to-face contact. For example, rather than read a form over a web-based platform, therapists have gone to a family member's home and dropped the documents into a mailbox or in front of their door. Therapists have also used available agency resources to increase contact. For example, therapists have dropped off care packages with food, clothing, and other essential items (e.g., diapers, toys, blankets). These accommodations were only possible because of the organizational and leadership drivers at the community agencies. The leaders at agencies developed plans to maintain safe social distancing guidelines for inperson contact, provide resources for necessary personal protective equipment, and secure care packages essential for families.

The loss of opportunities for in-person contacts with difficult-to-engage family members required multiple strategies to facilitate an initial conversation. Therapists called from various numbers, coordinated reaching out jointly with the referral sources, used text, email, and standard mail, and used e-cards sent via email or text.

Another area of clinical guidance was imparting the need for therapists to revisit the focus and purpose of strategies to engage family members in the treatment. Therapists had to recognize that this was a process rather than a discrete event that occurred through a single contact with a family member(s). For example, it was vital that therapists carefully and systematically work through the engagement process. Multiple contacts with different family members were appropriate, especially when the therapist or agency carried significant safety concerns. For example, in families with suspected or known intimate partner violence cases, therapists were directed to create opportunities for engaging in individual conversations with each family member. This direction was quite complicated, given that family members were often quarantined in the same household. Some agencies/systems permitted face-to-face contacts following approved social distancing and safety measures (e.g., initial health screen for symptoms, masks, the distance between therapists and family members), and therapists were able to create space for individual discussions.

One of the biggest challenges was ensuring that family members had access to materials that therapists used in sessions. Mailing material or sharing screens were simple and effective solutions in many circumstances. Again, many therapists used this as an opportunity to create additional face-to-face contacts by going to the family home to drop off the materials. For example, in one high-risk family referred from the child welfare system, the therapist put the behavior change packet on the family's porch and had all family members come outside to say hello from an appropriate distance. Most families appreciated this type of contact, mainly since many had minimal social contact.

In general, therapists initially viewed the tele-session medium as a barrier to introducing, practicing, and developing new skills or behaviors. However, through the sharing of strategies and ideas in the "Lessons Learned" document and training and coaching, therapists discovered how technology could enhance this process. For example, over time, therapists reported finding many creative ways to involve family members in tasks in sessions, such as using whiteboards or other features available on most tele-platforms, playing clips available online to illustrate a key concept or skill, and using memes and other visuals to make the sessions more engaging and lively.

\section{Comparing Service Profiles and Clinical Outcomes in 2019 and 2020}

To understand the potential impact of the shift to tele-services, FFT LLC compared information on clinical processes and outcomes from 2020 to the same period during 2019. 
For these comparisons, data from the web-based CSS were used. The CSS captures session contacts and notes, assessments (pre and post, process, and outcome), and model performance (through fidelity and dissemination adherence ratings), as well as therapist and client reported outcomes. The CSS was upgraded in March 2020 to enhance reporting

Table 2 Demographics comparison of 2019 and 2020

\begin{tabular}{|c|c|c|c|c|}
\hline \multirow[t]{2}{*}{ Variable } & \multicolumn{2}{|l|}{2019} & \multicolumn{2}{|l|}{2020} \\
\hline & $n$ & $\%$ & $n$ & $\%$ \\
\hline Cases Closed & 8820 & & 7728 & \\
\hline \multicolumn{5}{|l|}{ Gender } \\
\hline Male & 5007 & 56.7 & 4374 & 56.6 \\
\hline Female & 3775 & 42.8 & 3299 & 42.6 \\
\hline Other & 38 & 0.05 & 55 & 0.08 \\
\hline \multicolumn{5}{|l|}{ Race/ethnicity } \\
\hline White & 3459 & 39.2 & 3355 & 43.4 \\
\hline Black & 2717 & 30.8 & 1932 & 25.0 \\
\hline Hispanic/Spanish & 1400 & 15.8 & 1028 & 13.3 \\
\hline Bi-racial & 478 & 5.41 & 425 & 5.49 \\
\hline Other & 360 & 4.00 & 412 & 5.33 \\
\hline Asian & 134 & 1.51 & 145 & 1.87 \\
\hline Native American/Alaskan & 128 & 1.45 & 79 & 1.02 \\
\hline Maori & 119 & 1.34 & 60 & 0.77 \\
\hline Native Hawaiian/Isl Pacific & 22 & 0.24 & 52 & 0.67 \\
\hline Aboriginal & & & 72 & 0.93 \\
\hline Torres Strait Islander & & 2 & & $<.01$ \\
\hline Unknown & 3 & $<0.01$ & 166 & 2.14 \\
\hline
\end{tabular}

Case Closed includes all closed cases from March 15 to September 1 each year

Other includes Middle Eastern/North African

Aboriginal and Torres Strait Islander were not included as subcategories in the 2019 version of the CSS capabilities. Therapists entered all new cases into this system while completing cases in the old CSS version. The data from both systems were collected from March 15, 2020, to September 1, 2020. For comparison purposes, the same time period was used to pull data from the CSS's old version for 2019. The shift to teletherapy occurred around March 15. At this time, therapists entered their session notes as usual; however, by the end of March, FFT, LLC updated the CSS to allow for the choice of tele-sessions. It should be noted that the Institutional Review Board of the organization the lead author is affiliated with reviewed the project and determined that this project did not require IRB oversight because this was a program evaluation that did not involve testing hypotheses or a new intervention approach.

From March through the beginning of September 2020, FFT therapists provided over 35,000 tele-sessions to over 11,000 youth and families, and they continue to do so at the time of this report. Table 2 provides descriptive data on the closed families from March 15 to September 1 in 2019 and 2020. Overall, the categories of gender (approximately $43 \%$ female; $57 \%$ male in both years), age (16 in 2019, 15 in 2020), and race/ethnicity suggest little differences between the populations served during the two time periods.

The upper section of Table 3 (Case Closure) displays information on all cases that closed during the March 15 to September 1 time period in 2019 and 2020. There are two possible reasons for closure in FFT. First, the youth and family prematurely leave the service; hence they have not completed the model. Some of the more common causes are engagement failure after at least one session, youth incarceration or placement, or youth runaway behavior. Second, the youth and family complete all five phases of the model, with reductions in risk factors and improvement in protective factors.
Table 3 Case closure and case tracking comparison of 2019 and 2020

\begin{tabular}{|c|c|c|c|c|c|c|}
\hline \multirow[t]{2}{*}{ Variable } & \multicolumn{3}{|l|}{2019} & \multicolumn{3}{|l|}{2020} \\
\hline & $n$ & $\%$ & Avg. & $n$ & $\%$ & Avg. \\
\hline Number of closed cases ${ }^{\mathrm{a}}$ & 6411 & & & 5460 & & \\
\hline Closed due to non-completion & 1345 & 20.9 & & 1375 & 25.2 & \\
\hline Completed FFT & 5066 & 79.1 & & 4085 & 74.8 & \\
\hline \multicolumn{7}{|l|}{ Case tracking } \\
\hline Contacts & 79,316 & & 8.99 & 80,836 & & 7.31 \\
\hline Face-to-face sessions & 54,462 & 100 & & 14,627 & 37.3 & \\
\hline Tele-sessions & 0 & 0 & & 24,604 & 62.7 & \\
\hline Average number of sessions & & & 13.5 & & & 13.6 \\
\hline $\begin{array}{l}\text { Average number of days in service } \\
\text { for completed cases }\end{array}$ & & & 146 & & & 131 \\
\hline
\end{tabular}

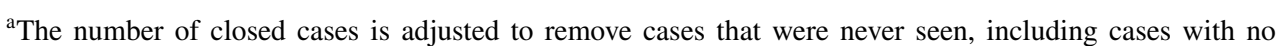
accurate contact information, families that declined services, and the youth's whereabouts are unknown. Cases that were closed outside of therapist control were also removed (e.g., the family moved out of the area, losing funding) 
As shown in Table 3, the overall completion rate was higher in 2019 than in 2020 (79\% vs. 75\%, respectively). FFT national standards for the within therapist control range from 70 to $80 \%$ and both years are well within the national average. It should be noted that there was a high percentage of new referrals in both samples, but it was particularly high in 2020 (51\%) as new sites started FFT training. This growth is relevant to evaluating completion rates because many new referrals were still active after September 1 and still have an opportunity to complete treatment. As such, it is likely that early dropouts among these new cases disproportionately influenced the completion rate in 2020.

The lower section of Table 3 (Case Tracking) reflects the number of contacts, sessions, and tele-sessions for the two time periods. A contact is any collateral activity supporting model delivery, such as phone calls, meetings, and court appearances. Contacts generally occur throughout the five phases but have consistent increases during early and later model phases. Sessions are face-to-face clinical sessions with at least one caregiver and youth. A tele-session is a session that is provided via a virtual format.

The number of contacts was similar; however, on average more contacts occurred in 2019 than in 2020 (8.99 vs. 7.31 ). The table reflects a $73 \%$ decrease in the number of face-to-face sessions from 2019 to 2020 and a $100 \%$ increase in tele-sessions. Despite the pandemic, there were still some face-to face sessions occurring; however, the majority, or $63 \%$ of the sessions provided, were through virtual format.

The program's average days are tracked as part of FFT, LLC's quality assurance and quality improvement program. The national standard is 60 to 180 days for FFT and 60 to 240 days for FFT-CW ${ }^{\circledR}$. Both years reflect the meeting of these standards. For example, the average number of days in service for completed cases was 146 in 2019 and 131 in 2020.

Table 4 examines the outcomes for the cases that were closed for the two years. FFT LLC collects information from therapists on specific ultimate outcomes, including the number of youth who remain in the community and with family, are in school or working, and have committed no new law violations at closure. At the time of closure, therapists also complete a Therapist Outcome Measure (TOM). Data were collected to demonstrate therapist outcome consistency. The TOM is a brief questionnaire that therapists complete after the family has been closed, and it is completed for all cases, irrespective of whether they completed treatment. The TOM reflects the therapist's perspective of family change, improvements in communication, youth behavior, parenting, parental supervision, and conflict level. In 2020, the TOM was expanded to include questions regarding specific youth referral behaviors, such as drug and alcohol use, school attendance and performance, and runaway and illegal behavior. The measure uses a 6-point Likert scale with
Table 4 Outcomes Comparison for 2019 and 2020

\begin{tabular}{|c|c|c|c|c|}
\hline \multirow[t]{2}{*}{ Variable } & \multicolumn{2}{|c|}{2019} & \multicolumn{2}{|c|}{2020} \\
\hline & $\%$ & Avg. & $\%$ & Avg. \\
\hline \multicolumn{5}{|l|}{ Ultimate outcomes } \\
\hline Youth remaining at home in community & 98 & & 98 & \\
\hline Youth attending school/GED/working & 95 & & 95 & \\
\hline Youth with no new law violations & 90 & & 92 & \\
\hline \multicolumn{5}{|l|}{ Therapist outcome measure $(\text { goal } \geq 3)^{\mathrm{a}}$} \\
\hline Q1 Improvement in family relationships & & 3.82 & & 3.79 \\
\hline Q2 Improvement in communication & & 3.77 & & 3.82 \\
\hline Q3 Improvement in youth behavior & & 3.66 & & 3.64 \\
\hline Q4 Improvement in parenting & & 3.66 & & 3.66 \\
\hline Q5 Improvement in youth supervision & & 3.62 & & 3.55 \\
\hline Q6 Reduction of conflict & & 3.78 & & 3.82 \\
\hline \multicolumn{5}{|l|}{2020 New additional questions } \\
\hline Q7 Reduction in illegal behavior & & & & 4.13 \\
\hline Q8 Reduction in runaway behavior & & & & 4.19 \\
\hline Q9 Increase in school attendance & & & & 3.60 \\
\hline Q10 Improvement in school performance & & & & 3.55 \\
\hline Q11 reduction in alcohol use & & & & 3.91 \\
\hline Q12 reduction in drug use & & & & 3.83 \\
\hline \multicolumn{5}{|l|}{ FFT clinician model performance ratings } \\
\hline Average fidelity (goal $\geq 3$ ) & & 3.77 & & 3.94 \\
\hline $\begin{array}{l}\text { Average dissemination adherence } \\
(\text { goal } \geq 4)\end{array}$ & & 4.37 & & 4.40 \\
\hline
\end{tabular}

${ }^{\text {a }}$ Scores on this measure range from 0 (worse) to 5 (very much improved)

scores ranging from 0 (worse) to 5 (very much improved). Predictive validity of the TOM has been shown in prior research (Turner et al., 2019).

The outcomes reported by therapists for youth remaining in the home (98\% both years) and school (95\% both years) and the percentage of youth with new law violations (90\% vs. 92\%) were consistent in 2019 and 2020. These rates are consistent with the FFT LLC standard of maintaining over $85 \%$ success in these categories. The therapists' ratings for other outcomes were consistent in 2019 and 2020, with scores over 3.5 for all items, including the new items added in 2020. All scores were above the FFT LLC minimum standard of three or higher.

The final area of comparison is therapist fidelity and dissemination adherence. The assessment of therapist fidelity involves systematic tracking of therapist implementation through formal group case staffing and individual supervision sessions, and other informal contacts. Following every clinical staffing, the clinical supervisor completes a fidelity rating for each case reviewed for each therapist. This fidelity rating reflects the degree of clinical adherence and competence for that therapist's work in that case in a specific session. Ratings are completed using Likert scales and 
producing an overall rating by combining the scores to create a final score that ranges from 0 (lowest) to 6 (highest). The therapist dissemination adherence addresses the extent to which the therapist is complying with the required administrative documentation (using the web-based tracking system) and the basic parameters of implementation (e.g., flexibility and intensity in scheduling, contact with key systems). Ratings are based on a 7-point Likert-type scale ranging from 0 (none) to 6 (always).

The results for therapist fidelity were consistent in 2019 and 2020 (3.77 and 3.94, respectively). In both years, the average fidelity rating exceeded the FFT LLC standard for fidelity of 3.0. The dissemination ratings were also consistent in 2019 and 2020 (4.37 and 4.40, respectively) and exceeded the FFT LLC standard of 4.0.

\section{Summary and Lessons Learned}

Shifting training, consultation, and clinical practice guidelines was a monumental challenge. As with any system with long-standing relationships, the system's expected tendency is to resist new recommendations and drift back to the established, familiar patterns. However, the intensity of the COVID-19 crisis led to an immediate shared experience that served as a foundation for collaboration that crashed through the tendency to maintain homeostasis and created a context of openness to do whatever it takes to serve youth and families. Overall, systems and agencies were open to recommendations and guidance about shifting training from inperson to webinar formats. Initially, one large system pushed back on the mid-March 2020 recommendation to suspend all in-person training to the end of April 2020 but ultimately agreed when their city's infection rates sharply increased. In this paper, the implementation framework articulated by Fixsen et al., 2005) was used to provide a coherent summary of the types of accommodations/innovations that were made to support the implementation of FFT in the early stages of COVID-19. Specifically, this paper described the organizational and leadership drivers (i.e., technical and adaptive leaders) critical in ensuring competent delivery of FFT via tele-sessions during the early stages of COVID-19.

Reports from FFT LLC expert trainers, experts, therapists, and supervisors from community-based partners have been overwhelmingly positive. Aspects of the webinar format have been beneficial, such as using multi-media during training, sharing multiple screens, scheduling training, and decreased travel-related costs. Nonetheless, the feedback has been that the experiential, relationship-building aspects of in-person training are more challenging to replicate in a virtual webinar space. Likewise, role plays and practicing skills do not have the same intensity and learning potential in a webinar format.
Concerning practice, the shift to tele-sessions was necessary for FFT to remain a viable clinical alternative. Agencies were as much of a driver in pushing for this shift as FFT LLC. As noted above, a significant push for this shift was financial. Sites needed definitive recommendations from a developer to ensure that they would be reimbursed for clinical activities. The flexibility that systems and funding bodies demonstrated during this time was exceptional. Again, with few exceptions, systems were flexible in creating new guidelines and accepting exceptions to their standard rules of practice to pave the way for the delivery of tele-sessions, for example, accepting various communication platforms to increase the accessibility and viability of tele-services for youth and families.

The guiding focus was on implementing FFT with integrity to its core principles and techniques. This focus forced all conversations back to principles and what FFT might look like throughout treatment. Ironically, this refocusing on the core principles helped strengthen knowledge about the model for FFT LLC experts and community-based therapists and supervisors.

From March 15, 2020, to September 1, 2020, over 11,000 families were seen, and over 35,000 tele-sessions were conducted. This activity represented a remarkable shift in global practice. More importantly, the descriptive comparison of closed cases during the same period in 2019 suggests that delivering FFT via tele-services is a viable alternative. Similar rates were observed in 2019 and 2020 for treatment completion ( $79 \%$ vs. $75 \%$ ), the number of sessions (13.5 vs. 13.6), and therapist fidelity (3.77 vs. 3.94 ). Also, therapists reported comparable outcomes in 2019 and 2020 for youth that remained in the home (98\% both years), in school (98\% both years), and had no new law violations ( $90 \%$ in 2019 , $92 \%$ in 2020). These findings were consistent with the therapist's reported outcomes on multiple variables. It should be noted, however, that these results do not suggest equivalency. The comparisons were a non-scientific program evaluation that did not include formal hypotheses and analyses.

\section{Lessons Learned}

The unprecedented issues in 2020 demanded innovative solutions to ensure that families continued to receive highquality, competently delivered FFT services. Although the accommodations required were unique to the circumstances surrounding COVID-19, many of these innovations are broadly relevant for the future implementation of established in community settings.

Consistent with the implementation drivers articulated by Fixsen et al. (2005), flexible and adaptive leadership is required to be able to respond to complex circumstances. Leadership that understands the core elements of the practice model is critical for ensuring that the innovations 
re-implemented in a manner that does not diminish therapists' ability to practice the models with high fidelity. Leadership also needs to be consistent and decisive while remaining open to feedback. The development of communication channels between therapists, administrators, and training organizations creates an opportunity to identify and respond to challenges in relevant and potentially effective ways.

Developing leadership and communication processes is an aspect of implementation that should be attended to at every stage of implementation, not just in the face of a crisis. Training organizations, like FFT LLC, systematically focus on developing leadership skills throughout implementation to ensure that local agencies and systems have the requisite skills sets to implement FFT with fidelity. One aspect of this process involves nurturing collaborative relationships between training organizations and agency administrators. Similarly, agency leaders are also encouraged to develop and nurture strong ties with community stakeholders. Such community-level relationships provide a foundation for being able to develop innovative solutions during a crisis. For example, it was consistently observed that systems with strong ties between community agencies and community stakeholders could rapidly develop innovative solutions for referring families to services and linking families to external resources when necessary. In contrast, systems with weaker pre-COVID-19 links between community agencies and community stakeholders tended to struggle with referral processes, managing high-risk families, and helping families get access to necessary resources and services.

Maintaining strong links between training organizations and community agencies also helped ensure that innovations and leadership decisions were shared and that solutions were implemented in a manner that maintained fidelity. For example, FFT LLC leaders led the development of innovations to clinical implementation. They provided additional coaching about implementing the clinical model using tele-sessions. At the same time, agency leaders (a) created resources and removed barriers for delivering tele-services and (b) developed and implemented innovative plans together with community stakeholders.

\section{Limitations of this Overview}

This paper reviews the primary authors' experiences collated from documents and conversations with FFT LLC experts and community partners over the past six months. As such, this paper did not include a formal qualitative or quantitative evaluation framework. The information is purely descriptive and based on the individual and shared perspectives of the co-authors. Before 2020, FFT LLC worked to develop a significant upgrade to its web-based CSS platform to document all aspects of service delivery. Throughout the first six months of 2020, FFT LLC rolled out the revisions to thousands of therapists nationally and internationally. The company is still in the process of fine-tuning this system and working out glitches. At this time, formal analyses are not possible since the data are still being reviewed and cleaned for accuracy. Finally, all of the outcomes were based on therapist reports. Future research must include family reports and objective indicators from juvenile justice and child welfare databases.

\section{Declarations}

Conflict of interest Drs. Robbins and Midouhas receive compensation from FFT LLC, an organization that provides training and consultation services to disseminate Functional Family Therapy.

\section{References}

Alexander, J. F., \& Parsons, B. V. (1982). Functional Family Therapy: Principles and procedures. Brooks \& Cole. https://doi.org/10. 1037/11621-000

Alexander, J. F., Waldron, H. B., Robbins, M. S., \& Neeb, A. A. (2013). Functional family therapy for adolescent behavior problems. American Psychological Association. https://doi.org/10.1037/ 14139-000

Bertram, R. M., Blase, K. A., \& Fixsen, D. L. (2015). Improving programs and outcomes: Implementation frameworks and organization change. Research on Social Work Practice, 25(4), 477-487. https://doi.org/10.1177/1049731514537687

Darnell, A. J., \& Schuler, M. S. (2015). Quasi-experimental study of Functional Family Therapy effectiveness for juvenile justice aftercare in a racially and ethnically diverse community sample. Children and Youth Services Review, 50, 75-82. https://doi.org/ 10.1016/j.childyouth.2015.01.013

Fixsen, D. L., Naoom, S. F., Blase, K. A., Friedman, R. M., \& Wallace, F. (2005). Implementation research: A synthesis of the literature. University of South Florida, Louis de la Parte Florida Mental Health Institute, The National Implementation Research Network.

Functional Family Therapy. (2021, January, 15). FFT research. https:// www.fftllc.com/about-fft-training/fft-research.html.

Gottfredson, D. C., Kearly, B., Thornberry, T. P., Slothower, M., Devlin, D., \& Fader, J. J. (2018). Scaling-up evidence-based programs using a public funding stream: A randomized trial of Functional Family Therapy for court-involved youth. Prevention Science, 14, 939-953. https://doi.org/10.1007/s11121-018-0936-Z

Henggeler, S. W., Pickrel, S. G., \& Brondino, M. J. (1999). Multisystemic treatment of substance abusing and dependent delinquents: Outcomes, treatment fidelity and transportability. Mental Health Service Research, 1, 171-184. https://doi.org/10.1023/A:10223 73813261

Henggeler, S. W., Schoenwald, S. K., Borduin, C. M., Rowland, M. D., \& Cunningham, P. B. (2009). Multisystemic therapy for antisocial behavior in children and adolescents. (2nd ed.). Guilford Press.

Robbins, M. S., Alexander, J. F., Turner, C. W., \& Hollimon, A. (2016). Evolution of Functional Family Therapy as an evidence-based practice for adolescents with disruptive behavior problems. Family Process, 55, 543-557. https://doi.org/10.1111/famp.12230

Turner, C. W., Robbins, M. S., Rowlands, S., \& Weaver, L. R. (2017). Summary of comparison between FFT-CW ${ }^{\circledR}$ and Usual Care from Administration for Children's Services. Child Abuse and Neglect, $69,85-95$ 
Turner, C. W., Robbins, M. S., Winokur Early, K., Blankenship, J. L., \& Weaver, L. R. (2019). Juvenile justice risk factors and Functional Family Therapy fidelity on Felony Recidivism. Criminal Justice and Behavior, 46, 697-717. https://doi.org/10.1177/0093854818 813184
Yuan, C. T., Nembhard, I. M., Stern, A. F., Brush, J. E., Krumholz, H. M., \& Bradley, E. H. (2010). Blueprints for the dissemination of evidence-based practices in health care. The Issue Brief (Commonwealth Fund), 86, 1-16 